\title{
HPV-Impfung: hohe Effektivität nach vier Jahren
}

\section{Die Impfung gegen humane Papillomaviren (HPV) ist seit mehreren Jahren etabliert. Mittlerweile liegen die Langzeitergebnisse zweier großer Studien mit über 17.000 Frauen vor.}

$\mathrm{n}$ zwei großen randomisierten, placebokontrollierten Studien (FUTURE I und FUTURE II) erhielten 17.622 Frauen (16-26 Jahre) drei Dosen einer Vakzine gegen die HPV-Serotypen 6, 11,16 und 18 oder Placebo. Ausgeschlossen waren Frauen mit früheren auffälligen Abstrichen, mit mehr als vier Sexualpartnern im Leben sowie Schwangere. Bereits zu Studienbeginn waren $15 \%$ der Frauen positiv für mindestens einen der im Impfstoff enthaltenen Serotypen. Nach 42 Monaten wurde die Impfeffektivität für das Auftreten von niedriggradigen Läsionen und Kondylomen bestimmt.

Bei den Frauen, die zu Beginn der Studie ohne Nachweis von HPV 6, 11, 16 und 18 waren, lag die Effektivität bezüglich zervikaler intraepithelialer Neoplasien ersten Grades (CIN 1), die positiv für diese Serotypen waren, bei $96 \%$. Für intraepitheliale Neoplasien gleichen Grades von Vulva und Vagina betrugen die Werte jeweils $100 \%$, für Kondylome $99 \%$.

Bei zu Studienbeginn HPV-negativen Frauen ergaben sich bezüglich aller HPVSerotypen als Auslöser von Läsionen folgende Impfeffektivitäten: $30 \%$ (CIN 1), 75 bzw. 48\% (intraepitheliale Neoplasien von Vulva und Vagina) sowie 83\% (Kondylome).

Die dargestellten Ergebnisse betreffen vollständig geimpfte Studienteilnehmerinnen (Per-Protocol-Analyse). Die Effektivitätsraten aller eingeschlossenen Frauen in der Intention-to-Treat-Analyse lagen teilweise deutlich niedriger.

Fazit: Die Autoren sehen in den Ergebnissen der Langzeitbeobachtung die

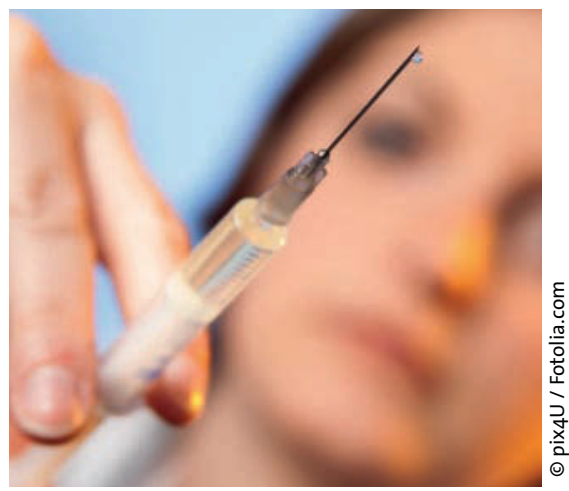

Ein Piks gegen HPV scheint zumindest niedriggradige Läsionen und Kondylome zu verhindern.

Schutzwirkung der HPV-Impfung gegen niedriggradige Läsionen und Kondylome bestätigt.

af

FUTURE I/II Study Group. Dillner J et al. Four year efficacy of prophylactic human papillomavirus quadrivalent vaccine against low grade cervical, vulvar, and vaginal intraepithelial neoplasia and anogenital warts: Randomised controlled trial.

Br Med J 2010; 340: C3493

\section{Wie hoch ist die diagnostische Sicherheit der Teledermatoskopie?}

Die Diagnosestellung via Teledermatoskopie erzielt diversen Studien zufolge annähernd gleiche diagnostische Genauigkeit wie die direkte Untersuchung. Oft wurde der Vergleich allerdings mit befundenden Ärzten derselben Klinik gemacht. Und man untersuchte häufig vorselektierte, meist melanozytäre Läsionen.

n dieser internationalen Studie wurde besonders streng geprüft, wie fehleranfällig es ist, die Epilumineszenz-Mikroskopie telemedizinisch zu nutzen: Man analysierte die Abweichungen in der Auswertung durch Untersucher auf verschiedenen Kontinenten. Erfasst und mit der Diagnose-Software („MoleMap“) gespeichert wurden 979 Läsionen von 206 Patienten. Die Aufnahmen wurden fünf erfahrenen Dermatologen aus Australien, USA und Neuseeland zugesandt.

Was Melanome und benigne Nävi betraf, kamen vier der fünf Fachärzte (,A“ bis „D“) zu weitgehend gleichen Auswertungen. Der fünfte Untersucher
(„E“) diagnostizierte deutlich mehr Melanome und atypische Nävi.

Die Autoren führen diese Abweichung auf mögliche Unterschiede in den Definitionen zurück - über die Konfusionen, die der Terminus ,atypischer Nävus“ stiftet, wurde in der Vergangenheit schon häufig diskutiert. Hinzu kommt möglicherweise eine mangelnde Vertrautheit mit der Studienpopulation: Diese umfasste Patienten eines neuseeländischen Zentrums. Und die Dermatologen A-D entstammten alle der südlichen Hemisphäre, E der nördlichen.

Bei den Basalzellkarzinomen und den seborrhoischen Keratosen gab es kaum unterschiedliche Beurteilungen; stärker waren die Schwankungen bei den Diagnosen In-situ-Spinaliom und aktinische Keratose. Bezüglich des invasiven spinozellulären Karzinoms (SCC) schließlich differierten die Interpretationen erheblich.

Fasste man allerdings die Diagnosen in drei Kategorien zusammen, nämlich: atypische und benigne Nävi, aktinische Keratosen und SCC in situ (also Präkanzerosen und In-situ-Karzinome) sowie maligne Läsionen (also Melanome, Basaliome und invasive SCC), so lagen die Diagnosen deutlich näher beieinander. D.h., alle Dermatologen stimmten in hohem Maß darin überein, welche Hautveränderungen mit hoher Wahrscheinlichkeit maligne waren $(K=0,57-0,93)$.

Fazit: Nach diesen Daten ermöglicht es die Teledermatoskopie, sicher zwischen malignen und benignen Läsionen zu unterscheiden.

wpa

Tan E et al. Interobserver variability of teledermoscopy: an international study. Br J Dermatol 2010; 163: 1276-81 